\title{
A survey of healthcare professionals' treatment of patients with eating disorders and obesity in Saudi Arabia, and the availability of specialised resources
}

\section{Amnah Alsabbah ( $\sim$ a_dit@hotmail.com )}

\section{Research article}

Keywords: Eating disorders, Saudi Arabia, obesity, anorexia, bulimia nervosa, stigma, mental health, body image

Posted Date: May 22nd, 2020

DOI: https://doi.org/10.21203/rs.3.rs-30264/v1

License: (c) (i) This work is licensed under a Creative Commons Attribution 4.0 International License.

Read Full License 


\section{Abstract}

Background: In developing countries (DCs) such as Saudi Arabia (SA), previous studies have considered EDs to be rare and related to westernised culture, where a thin physique is idolised. However, the rapid transition in lifestyles in DCs makes it reasonable to hypothesise that ED prevalence may be rising in these countries. Similarly, studies in SA show that obesity levels are increasing. However, studies that include the role of psychology for obesity management or in the assessments for metabolic surgeries (MS) are lacking. These deficiencies urge to measure this phenomenon in SA and develop additional management strategies for these disorders. A survey was designed to evaluate the number of referrals for EDs and obesity, the management and level of availability of ED specialists as reported by health care staff and the use of mental-health services in treating EDs and obesity.

Methods: A total of 15 health services in SA were surveyed and a total of 123 survey questionnaires were completed by health professionals. Participants were asked to respond to a written survey that included questions regarding the number of referrals for EDs and obesity and available treatment as well as body mass index criteria for MS and the number of patients referred for these procedures.

Results: Findings from this study add to those from a few previous studies revealing that all EDs are equally prevalent in diverse cultures. Additionally, this survey showed that the obesity rate is high, however, there is limited psychological interventions in this regard.

Conclusion: The perspective of narrowing the cultural categories to either western or non-western countries when considering the aetiology of EDs without considering other socioeconomic factors needs to be changed. There is a requirement for specialised ED training, a need to identify a conceptual framework to provide evidence-based management and increase the levels of involvement of psychiatric professionals in the management of obesity. Although this study was conducted in one region, the results should be generalisable to other areas and to other non-westernised countries were EDs are considered rare. Key words: Eating disorders, Saudi Arabia, obesity, anorexia, bulimia nervosa, stigma, mental health, body image.

\section{Plain English Summary:}

Over the last few decades, the Kingdom of Saudi Arabia (KSA) has experienced rapid socio-cultural changes caused by the accelerating economy of the Arabian Gulf region. The prevalence of obesity among adults in Saudi Arabia has been increasing. This study aims to identify the number of referrals for Eating Disorders (ED) and obesity, the management and level of availability of ED specialists as reported by healthcare staff, and the use of mental health services in treating EDs and obesity. Although the result showed that the obesity rate is high. There is a requirement for specialised ED training, and increase the levels of involvement of psychiatric professionals in the management of obesity. 


\section{Abbreviations}

EDs Eating Disorders

ED Eating Disorder

DCs Developing Countries

SA Saudi Arabia

BN Bulimia Nervosa

AN Anorexia Nervosa

GPs General Practitioners

BED Binge Eating Disorder

BT Behavioural Therapy

CBT Cognitive Behaviour Therapy

BS Bariatric Surgery

MHSs Mental Health Services

MOH Ministry of Health

GHs General Hospitals

PSs Psychiatric Services

NICE.UK National Institute for Health and Care Excellence in the UK

DSM Diagnostic and Statistical Manual of Mental Disorders ICD Inter National Classification of Diseases

MDT Multi-Disciplinary Team

MS Metabolic Surgery DPs Dental Practitioners

RDs Registered Dieticians

EDNOS: Eating Disorder Not Otherwise Specified

BMI Body Mass Index

PC Primary Care 


\section{Introduction}

Early detection of eating disorders (EDs) is uncommon in SA. To date, only two studies have been carried out, one in the small city of Hail (Fatima, Fatima, \& Anwar, 2018) and the other in a secondary school (Alwosaifer, Alawadh, Wahab, Boubshait, \& Almutairi, 2018). There are no available studies among women in general hospitals or among women in mental health $(\mathrm{MH})$ facilities. Conversely, there has been a growing interest worldwide concerning EDs. For instance, in the UK, Micali et al. (2017) used a community-based sample of 5,658 participants recruited from the Avon longitudinal study, focusing on women in their 40 s and 50 s who met the criteria for 12-month to lifelong ED. It was found that $15.3 \%$ met the criteria for lifetime ED, while the 12-month prevalence of ED was 3.6\%. The work of Micali et al. (2017) is relevant to the current study for two main reasons. Additionally, in India, Srinivasan, Suresh, and Jayaram (1998) found that although the prevalence of EDs in Asian countries was lower than that in Western countries.

Apart from EDs, obesity is recognised as a worldwide health problem. Whether obesity is technically an ED is debatable; it is a chronic illness that can predispose to EDs, which are clearly medical disorders related to body image. Furthermore, the side effects of various medications related to targeted populations, such as antipsychotics and antidepressants, including Second-Generation Antipsychotics (SGAs), are associated with the risk of developing obesity rapidly in treated patients (Eum, Lee, \& Bishop, 2016). Obesity in KSA is far more prevalent than EDs. EDs affect approximately $3.8 \%$ of the population (Sabra, 2014), while obesity affects approximately 34.8\% (Al-Hazzaa, Abahussain, Al-Sobayel, Qahwaji, \& Musaiger, 2012). However, there are no available studies showing the prevalence of obesity in $\mathrm{MH}$ services.

The perception of weight varies from region to region in KSA; in some areas, being overweight is a symbol of prosperity and therefore consuming high-calorie food is the norm. In areas exposed to outside cultural influences, the exact opposite is true. Khalaf, Westergren, Berggren, Ekblom, and Al-Hazzaa (2015) found that less than one-quarter $(23.3 \%)$ of the participants had an accurate perception of their actual weight. This also correlated positively ( $p$ value 0.047 ) with high monthly incomes ( $>1300$ to $\$ 2650$ ), the number of cars in the household (3 or more compared to 2), and the type of residence (traditional house versus apartment) (Rasheed, 2014)

The present study area is heterogenous, seeking to provide recommendations that can be generalisable. The study objectives were to detect the number of referrals for EDs and obesity among women referred to mental health facilities. Also, Tit aimed to assess the levels of availability of specialised ED personnel and services to establish national recommendations. 


\section{Materials And Methods}

\section{Design}

This study was designed as a cross-sectional survey for the number of referrals for EDs and obesity reported by professionals working with these disorders

\section{Setting}

The study was conducted in Taif MH Hospital, Jeddah MH Hospital, King Abdul-Aziz MH Department, King Abdul-Aziz Hospital in Makkah, King Faisal Hospital, Heraa General Hospital, King Fahad Hospital, King Abdul-Aziz Hospital in Jeddah, Al-Noor Specialist Hospital, King Abdul-Aziz Specialist Hospital in Taif, King Abdullah Medical City, Aleskan, Alrusaifah, and Alawali General Practitioners (GPs).

\section{Sample}

The inclusion criteria of the study were healthcare professionals (psychiatrists, psychologists, physicians, family medicine physicians, Registered Dieticians (RDs), Dental Practitioners (DPs), and nurses working in mental health services and GHs; information about the frequency of referred adult women aged 18-65 from General Hospitals (GHs) to Psychiatric Services (PSs) and the number of received referrals in three Mental Health Services (MHSs) was obtained for the same age groups.

\section{Procedures}

A written survey was conducted among healthcare professionals. The survey was translated into Arabic in addition to the English language version for staff who had limited English language fluency. The researcher approached the medical directors in each service, then the head of the department for RD recruitment, psychologists and dental practitioners, the nursing director in each service, and the director of GPs, and requested to meet targeted staff to introduce the study and describe the written survey.

\section{Outcome Measures}

The outcome measure has four parts. The first covers socio-demographic data of the study participants: the healthcare professional name, profession, professional grade, hospital or service name, name of ward or unit (to avoid replicating the same cases), type of service (e.g., outpatient, day-patient, inpatient or community service) and work experience in years.

The second part clarifies information regarding the referred cases. Respondents were asked whether they had referred any patients with EDs/obesity to PS or received any referrals for patients with EDs/obesity in PS during their practice, and if so, approximately how many cases on average.

The third part of the questionnaire was dedicated to questions regarding the use of different treatments for these disorders and obesity, namely, individual psychotherapy, group therapy, family therapy, dietary advice, day-patient care. 
The fourth part of the scale is related to the care available, whether delivered by ED specialist staff or generally trained staff regarding obesity. Participants were asked about the number of obesity cases referred for BS (within the participant's period of practice) and for psychiatric assessment and rehabilitation pre-and post-surgery.

\section{Data analysis}

Information about the work experience of each professional, targeted BMI for BS referral and the number of referred cases for BS was compiled on a spreadsheet for descriptive statistical analysis using Excel 2016 for Windows, means \pm (SD).

\section{Ethical Considerations}

The study obtained ethical approval from the Ministry of Health in the Kingdom of Saudi Arabia and the selected hospitals. Participants were also asked to sign a consent form before enrolment in the study. Confidentiality of the participants was assured by giving them pseudonyms.

\section{Results}

A total of 15 services in the western region of SA were surveyed, of which 3 were MH services, 5 were general hospitals, 2 were specialised hospitals, 1 was a medical complex serving as one of the major referral and tertiary care centres for BS cases, and 3 were Primary Care (PC) service centres included to survey family medicine, a private dietetic centre and GPs. A total of 3,293 referrals were reported from all services. Of these, 2,409 (79.3\%) were from GHs, while PSs sections included only $884(20.7 \%)$ of the total. Questions were limited to women aged between 18 and 65 years with EDs or obesity who were referred to psychiatric services. Regarding medical records, no ED cases were documented between 21 June 2017and 20 July 2017 in all the researched services. Table 1 shows information about the participants.

\section{Please insert table 1 around here}

Determinants for referral and referral numbers by profession with bed numbers for all served hospitals (3 $\mathrm{MH}$ and 7 general hospitals), excluding GPs that did not include inpatient services, and the total referral numbers in each category for each profession are shown in Table 2. A total of $56.3 \%$ of the referrals were reported by RDs, $27 \%$ by psychiatrists (of which one case was for a member of the MHS staff), $>14.8 \%$ by GPs and family medicine consultants, $8.5 \%$ by DPs, $6.7 \%$ by physicians, $2.1 \%$ by nurses in PSs and $0.5 \%$ by nurses in GHs; only one referral was reported by a psychologist. The total referrals for each disorder were as follows: simple obesity comprised 79\%, obesity with EDs $13 \%$, AN 6.6\%, BED 5.3\% and BN 4.5\% of the cases. One obstetrics and gynaecology consultant reported that $5 \%$ of the females she worked with were diagnosed with pica, which is one of the EDNOS categories, and a dietetic consultant reported receiving referrals in percentages. 


\section{Please insert table 2 around here}

Table 3 shows proportions of professionals reporting available treatment for all disorders in psychiatric and general services, including a category named "other" that identifies other specific treatments for all disorders. All psychiatrists in the three MHSs reported that CBT was the type of individual therapy used most often in the treatment of these disorders because certain cognitive behaviours are the centre of the disease aetiology and this therapy has proven effective. Inpatient services were more common in the management of $\mathrm{AN}$ and $\mathrm{BN}$ but less common with regards to obesity.

\section{Please insert table 3 around here}

Table 4 shows the number of patients referred for bariatric surgery as well as BMI cut-off values (means \pm $\mathrm{SD}$ ). Most of the participants reported BMI as the main criterion for referring obese individuals for BS. However, only three participants reported BMI cut-offs for referral for BS as unknown, and one item was not answered. One was a GP with 2 years of experience, the second a psychiatrist with 18 years of experience, and the third a DPs with one year of experience. However, they reported referring 100, 120, and 2 patients, respectively, for bariatric surgery according to weight only (i.e. $>140 \mathrm{~kg}$ ).

\section{Please insert table 4 around here}

\section{Discussion}

This is the only study that has been conducted with the aim of assessing referrals received for EDs and obesity in psychiatric and GH and availability of specialised resources in KSA. Despite a substantial body of research worldwide, there is no available data regarding the prevalence of EDs, treatment strategies and treatment professionals in KSA, which was the driving force that motivated the researcher to conduct this survey.

The most important finding in this study is that in contrast to what was reported in previous studies, the result shows a considerable proportion of referrals for all EDs and reflects a worldwide prevalence of these disorders. However, obesity was the most common cause of referrals (78.9\%). Reported referrals received for various ED cases included $6.6 \%$ for $A N, 5.3 \%$ for $B E D$ and $4.5 \%$ for $B N$, while one obstetric gynaecology consultant reported that $5 \%$ of patients suffered from pica.

The total number of referrals for GHs and MHS equated to $29 \%$ of the total bed capacity in PSs and $71 \%$ of the total bed capacity in GHs. If all these cases were admitted for inpatient services, there is a strong need to treat this group of disorders by using services that are meant to provide treatment for a wide range of health complications. The average bed turnover rate in the GHs was $53.2 \%$ while in PSs, it was $5.2 \%$ (Al-Oklah \& Titi). Therefore, having SEDUs that can provide psychiatric management in a less stigmatising manner may be the best solution. Previous studies indicate that EDs are more prevalent in Western cultures. Additionally, EDs are far more common among women than men, reflecting cross- 
cultural differences in the quest for thinness for women (Allihaibi, 2015; Fatima et al., 2018; Keel \& Klump, 2003).

\section{Referrals for EDs and obesity from HCP}

Findings from this study similarly showed that in PSs, more than one-quarter of the total referrals were reported by psychiatrists whilst nurses reported $2 \%$ of the cases. Notably, all surveyed GHs, physicians and nurses reported nearly $7 \%$ and $0.5 \%$ of the total referrals, respectively; nonetheless the availability of psychiatrists and psychologists was not presented. In GP, where $14.8 \%$ of the total referrals were reported, family medical consultants provided psychoeducation for minor cases before referral to psychiatric services.

This classification makes it important to manage these disorders with a great emphasis on $\mathrm{MH}$. Nevertheless, the medical input is not compulsory but is required, depending on the severity of the case. Hence, it must be emphasised that treating these patients in PSs or Specialised Eating Disorder Units (SEDUs) is more beneficial when psychiatric input is appropriate for disorders of this nature. Another motive that supports treating ED patients in SEDUs relates to the need to maintain social development for patients and minimise isolation. In this regard, SEDUs can provide day-care programmes or community care that is deemed more beneficial, as it does not involve separating patients from their social environment.

However, this step could be achieved at a later stage, as currently, the emphasis on training current staff would be more realistic. Another finding from this study demonstrated the scarcity of the availability of psychologists; like the psychiatrists in GHs, they were only available in PSs. Psychologists reported only one case of ED; this number is paltry for a profession that plays a crucial role in the management of EDs and obesity.

This considerable difference in patient preferences may be attributable to several factors. Of primary importance is the stigma attached to seeking help from PSs, which are widely associated with treating individuals with mental illness. Therefore, eliminating the stigma that is often associated with mental disorders is of utmost importance and was discussed in the 2005 WHO European Ministerial Conference on $\mathrm{MH}$ in Helsinki. Unexpectedly, $\mathrm{MH}$ professionals who fight stigma could inadvertently be contributing to the stigma through the language they use (Puhl \& Suh, 2015).

\section{Results from obesity and BS survey}

Regarding BS, this study's findings showed that BMI was the main criterion for referring obese individuals for BS, which is the norm. The median for reported BMI cut-off values for BS referral was $>40$, with a median referral of four patients for each participant (Table 4), which agrees with the UK's NICE guidelines. Several participants did not report the BMI cut-off or responded to the item as "unknown". In the private dietetic units, only five patients were referred for BS. Referral for BS in the private dietetic centre had two 
criteria: either a BMl>50 or if nutritional therapy was not successful. The increase in movement towards modernised lifestyles that alter the way food is consumed, in combination with sedentary behaviours, are the most likely explanations of the high rate of obesity in SA. The high income of Saudi citizens allows many families to employee housemaids and nannies, decreasing energy expenditure for Saudi women.

Dinsa, Goryakin, Fumagalli, and Suhrcke (2012), in their systematic review, concluded that in low-income countries or in countries with a low human development index, the association between socio-economic status and obesity appears to be positive; the more affluent and/or those with higher educational attainment are more likely to be obese. Further legislation by the Saudi government has recently added a tax on sweetened drinks to reduce their consumption and consequently reduce obesity (Alsukait, Bleich, Wilde, Singh, \& Folta, 2020). 79\% of the reported referrals were due to obesity, while obesity in combination with EDs comprised $13 \%$ of the total cases. This exceeds the number in the UK, which recorded only 8,000 operations in 2011; between 2010 and 2013, only 18,000 BS operations were reported in the UK, even though the UK population is double that of SA (Welbourn et al., 2014).

\section{Management strategies for EDs and obesity}

Further analysis of the number of referrals for different professions is shown in Table 2. Other findings from this study showed that all three sub-diagnostic categories of ED are based on psychiatric management, which is the norm, while obesity and obesity with EDs were mostly managed by giving dietary advice. Furthermore, the survey results demonstrate the limited use of psychotherapies, such as Behavioural Therapy (BT) or Cognitive Behaviour Therapy (CBT), as a management strategy for obesity, as they were not popular in the surveyed services. CBT was reported as the most preferred intervention for obesity and BED (Castelnuovo et al., 2017). Thus, this approach may be beneficial when obesity occurs in combination with other EDs. Behavioural modification is important whatever the management of obesity. This was also suggested by Shah et al. (2020), who recommended that any treatment for obesity should be in combination with a structured behavioural modification programme.

\section{The availability of ED specialists}

Regarding the availability of ED specialists in psychiatric services, as indicated in Figure 1, the results from this study showed that over two-thirds of respondents $(68 \%)$ reported that ED treatment was provided by HCP who had general training in ED management; $4 \%$ reported that it was provided by ED specialists, and $22 \%$ gave no response. These results support the hypothesis that ED specialists are scarce in the region and there is a need for qualified professionals to provide specialised treatment for this population. 


\section{Findings from clinical nutritionists}

RDs received more than half of the referrals, which is considered a good indicator of the level of dietician engagement in treating these disorders. However, of the total number of surveyed RDs, only one was a member of staff in a psychiatric hospital, which is considered a limited staffing position for this profession. This lack of personnel may be the cause of the higher proportion of referrals received by dieticians in GHs than by dieticians in PSs, which indicates a need for more dieticians in PSs. RD are essential members in the MDT for ED management. Dietary restraints and abnormal eating behaviours constitute a basis for ED aetiology in combination with cognitive impairment. However, an RD is qualified to provide this knowledge at various levels of care (Wanner, Richard, Martin, Faeh, \& Rohrmann, 2017).

\section{Findings from primary care and GPs}

There is a need to focus on current HCP training and on how general health professionals, especially GPs and PC staff, can identify EDs, manage these patients and refer them in a more efficient way. Patients with EDs are typically first seen by their family physician. Ogg, Millar, Pusztai, and Thom (2013) suggested that patients with EDs consult their family physician more frequently than the control subjects in the study. Additionally, adequate knowledge of PC professionals is crucial in managing these patients. Patients perceived PCs as accessible and less stigmatising (Crawford et al., 2018) and psychiatrists in clinics reported that they can provide better quality of clinical care because of improved liaison with referring GPs (Strathdee, Williams, Shepherd, \& Wilkinson, 2014). However, this survey's findings showed that GPs and GHs do not include psychiatrists and psychologists and the role of providing psychiatric input is performed by the family medicine consultant before referral or through psychiatric counselling requests in medical words in GHs. Additionally, different ED screening tools can be used reliably in PC settings, such as SCOFF and EDE-Q, even though the latter was reported to perform better (GarciaCampayo et al., 2005; Mond, Robertson-Smith, \& Vitere, 2006). Studies have shown that early detection of EDs improves prognosis, which is an important target for these relapsing disorders (Nazzal, 2015).

\section{Findings from dental practitioners}

Compensatory behaviours, such as self-induced vomiting in some bulimic patients and oral hygiene in obese patients, affect dental health. Thus, dental practitioners such as dentists and oral hygiene specialists were interviewed regarding EDs and obesity referrals. As shown in Table 2, dental practitioners received $8.5 \%$ of the cases. The results of this study showed that most DPs receive patients diagnosed with EDs in their facilities. These patients are aware of the disorder but prefer not to seek assistance from PSs.

\section{Limitations and strengths of the study}

The study has some limitations as the first involved the posting and online surveying, as the response rate was low; unfortunately, only 4 respondents answered and returned the survey questionnaire. Indeed, many other methods could have been used to recruit participants for the study. However, due to time 
constraints, the author used only these methods. The second limitation was access to available staff. The time at which the study was conducted was a holiday season in SA; therefore, most staff were on vacation, which may have affected the participant recruitment.

Regarding strengths, personal interaction ensured truthful and high response rates in answering survey elements. Moreover, surveying staff from different professions (psychiatrists, psychologists, physicians, DPs, RDs, nurses) in all types of healthcare service provides a broader picture of the level of referrals as well as the level of awareness of these disorders and their available management. In addition, information from specific groups, such as professionals in PSs who received referrals for EDs and GH staff who might be the first HCP to encounter such cases, was very helpful.

\section{Conclusion}

The level of prevalence of any health problem has a major effect in determining and allocating human and monetary resources to address the problem. Moreover, many health problems such as EDs have received wide interest in the West due to their high incidence, while non-Western countries in the Middle East, particularly KSA.

\section{Declarations}

\section{Conflict of interest}

The author reports no conflict of interest.

\section{Acknowledgements}

The authors thank all healthcare professionals who contributed to the study.

\section{Ethical approval and consent to participate:}

N/A for participant but available for as a study approval in $\mathrm{MOH}$ facilities.

\section{Consent for publication:}

\section{N/A}

\section{Availability of data and materials:}

The datasets analyzed during the current study available from the corresponding author on reasonable request. 


\section{Competing interests:}

The author declare that she has no competing interests.

\section{Funding:}

All funding were by the author.

\section{Authors' contribution:}

The study has one author, and all contribution were made by Amnah Alsabbah.

\section{Acknowledgement:}

N/A

\section{References}

Al-Hazzaa, H. M., Abahussain, N. A., Al-Sobayel, H. I., Qahwaji, D. M., \& Musaiger, A. O. (2012). Lifestyle factors associated with overweight and obesity among Saudi adolescents. BMC public health, 12(1), 354.

Al-Oklah, H., \& Titi, S. SYLLABUS AND CONTENTS OF STAT1102 (1436/1437).

Allihaibi, M. M. (2015). Disordered eating attitudes among secondary schoolgirls in Al-Iskan sector, Makkah Al-Mukarramah, Saudi Arabia. International Journal of Medical Science and Public Health, 4(7), 939-946.

Alsukait, R., Bleich, S., Wilde, P., Singh, G., \& Folta, S. (2020). Sugary drink excise tax policy process and implementation: Case study from Saudi Arabia. Food Policy, 90, 101789.

Alwosaifer, A. M., Alawadh, S. A., Wahab, M. M. A., Boubshait, L. A., \& Almutairi, B. A. (2018). Eating disorders and associated risk factors among Imam Abdulrahman bin Faisal university preparatory year female students in Kingdom of Saudi Arabia. Saudi medical journal, 39(9), 910.

Castelnuovo, G., Pietrabissa, G., Manzoni, G. M., Cattivelli, R., Rossi, A., Novelli, M., . . Molinari, E. (2017). Cognitive behavioral therapy to aid weight loss in obese patients: current perspectives. Psychology research and behavior management.

Crawford, M. J., Sanatinia, R., Barrett, B., Cunningham, G., Dale, O., Ganguli, P., . . Lykomitrou, G. (2018). The clinical effectiveness and cost-effectiveness of lamotrigine in borderline personality disorder: a randomized placebo-controlled trial. American Journal of Psychiatry, 175(8), 756-764. 
Dinsa, G. D., Goryakin, Y., Fumagalli, E., \& Suhrcke, M. (2012). Obesity and socioeconomic status in developing countries: a systematic review. Obesity reviews, 13(11), 1067-1079.

Eum, S., Lee, A. M., \& Bishop, J. R. (2016). Pharmacogenetic tests for antipsychotic medications: clinical implications and considerations. Dialogues in clinical neuroscience, 18(3), 323.

Fatima, W., Fatima, R., \& Anwar, N. S. (2018). Prevalence of eating disorders among female college students of Northern Broader University, Arar, Kingdom of Saudi Arabia. International Journal of Child Health and Nutrition, 7(3), 115-121.

Garcia-Campayo, J., Sanz-Carrillo, C., Ibañez, J., Lou, S., Solano, V., \& Alda, M. (2005). Validation of the Spanish version of the SCOFF questionnaire for the screening of eating disorders in primary care. Journal of psychosomatic research, 59(2), 51-55.

Keel, P. K., \& Klump, K. L. (2003). Are eating disorders culture-bound syndromes? Implications for conceptualizing their etiology. Psychological bulletin, 129(5), 747.

Khalaf, A., Westergren, A., Berggren, V., Ekblom, Ö., \& Al-Hazzaa, H. M. (2015). Perceived and ideal body image in young women in south western Saudi Arabia. Journal of obesity, 2015.

Micali, N., Martini, M. G., Thomas, J. J., Eddy, K. T., Kothari, R., Russell, E., . . Treasure, J. (2017). Lifetime and 12-month prevalence of eating disorders amongst women in mid-life: a population-based study of diagnoses and risk factors. BMC medicine, 15(1), 12.

Mond, J., Robertson-Smith, G., \& Vitere, A. (2006). Stigma and eating disorders: is there evidence of negative attitudes towards individuals suffering from anorexia nervosa. Journal of Mental Health, 15, 519-532.

Nazzal, W. M. (2015). Body Perceptions and Weight Control Behaviors among An-Najah National University female Students, 2015.

Ogg, E. C., Millar, H. R., Pusztai, E. E., \& Thom, A. S. (2013). General practice consultation patterns preceding diagnosis of eating disorders. International Journal of Eating Disorders, 22(1), 89-93.

Puhl, R., \& Suh, Y. (2015). Stigma and eating and weight disorders. Current psychiatry reports, 17(3), 10.

Rasheed, P. (2014). Perception of body weight and self-reported eating and exercise behaviour among obese and non-obese women in Saudi Arabia. Public health, 112(6), 409-414.

Sabra, A. A. (2014). Obesity among female intermediate nursing students of health science collage in Dammam city, Saudi Arabia: prevalence and associated factors. Canadian Journal of Clinical Nutrition.

Shah, S. J., Borlaug, B. A., Kitzman, D. W., McCulloch, A. D., Blaxall, B. C., Agarwal, R., . . Gladwin, M. T. (2020). Research Priorities for Heart Failure With Preserved Ejection Fraction: National Heart, Lung, and 
Blood Institute Working Group Summary. Circulation, 141(12), 1001-1026.

Srinivasan, T., Suresh, T., \& Jayaram, V. (1998). Emergence of eating disorders in India. Study of eating distress syndrome and development of a screening questionnaire. International Journal of Social Psychiatry, 44(3), 189-198.

Strathdee, G., Williams, P., Shepherd, M., \& Wilkinson, G. (2014). Patterns of collaboration. Mental illness in primary care settings. London: Tavistock, 141-155.

Wanner, M., Richard, A., Martin, B., Faeh, D., \& Rohrmann, S. (2017). Associations between self-reported and objectively measured physical activity, sedentary behavior and overweight/obesity in NHANES 20032006. International journal of obesity, 41(1), 186-193.

Welbourn, R., Small, P., Finlay, I., Sareela, A., Somers, S., Mahawar, K., . . Kinsman, R. (2014). The United Kingdom national bariatric surgery registry. Second registry report, 2014.

\section{Tables}

Table 1: Information about participants' services.

\begin{tabular}{ccc} 
Professions & Number of professionals & $\begin{array}{c}\text { Working experience (years) } \\
\text { Mean } \pm(\text { SD) }\end{array}$ \\
\hline Psychiatrists & 20 & $12.05 \pm(7.4)$ \\
Psychologists & 7 & $1.6 \pm(1.8)$ \\
\hline Family medicine consultants/ GP & 16 & $10.1 \pm(10.2)$ \\
\hline Physicians & 8 & $3.25 \pm(193.03)$ \\
\hline Dental practitioners & 7 & $4.5 \pm(3)$ \\
\hline Dieticians & 35 & $2.8 \pm(1.6)$ \\
\hline Nurses (psychiatric services) & 21 & $7.6 \pm(7.3)$ \\
\hline Nurses (other services) & 9 & $3 \pm(2.8)$ \\
\hline Total & 123 &
\end{tabular}

Table 2: Referral frequency for each profession. 
Source of referral:

Number of consultation $\begin{array}{ccccccc}\text { Anorexia } & \text { Bulimia } & \text { Binge } & \text { Simple } & \text { Obesity with } & \text { Other } & \text { Total } \\ \text { Nervosa } & \text { Nervosa } & \text { Eating } & \text { Obesity } & \text { ED } & & \text { Cases }\end{array}$

By

profession

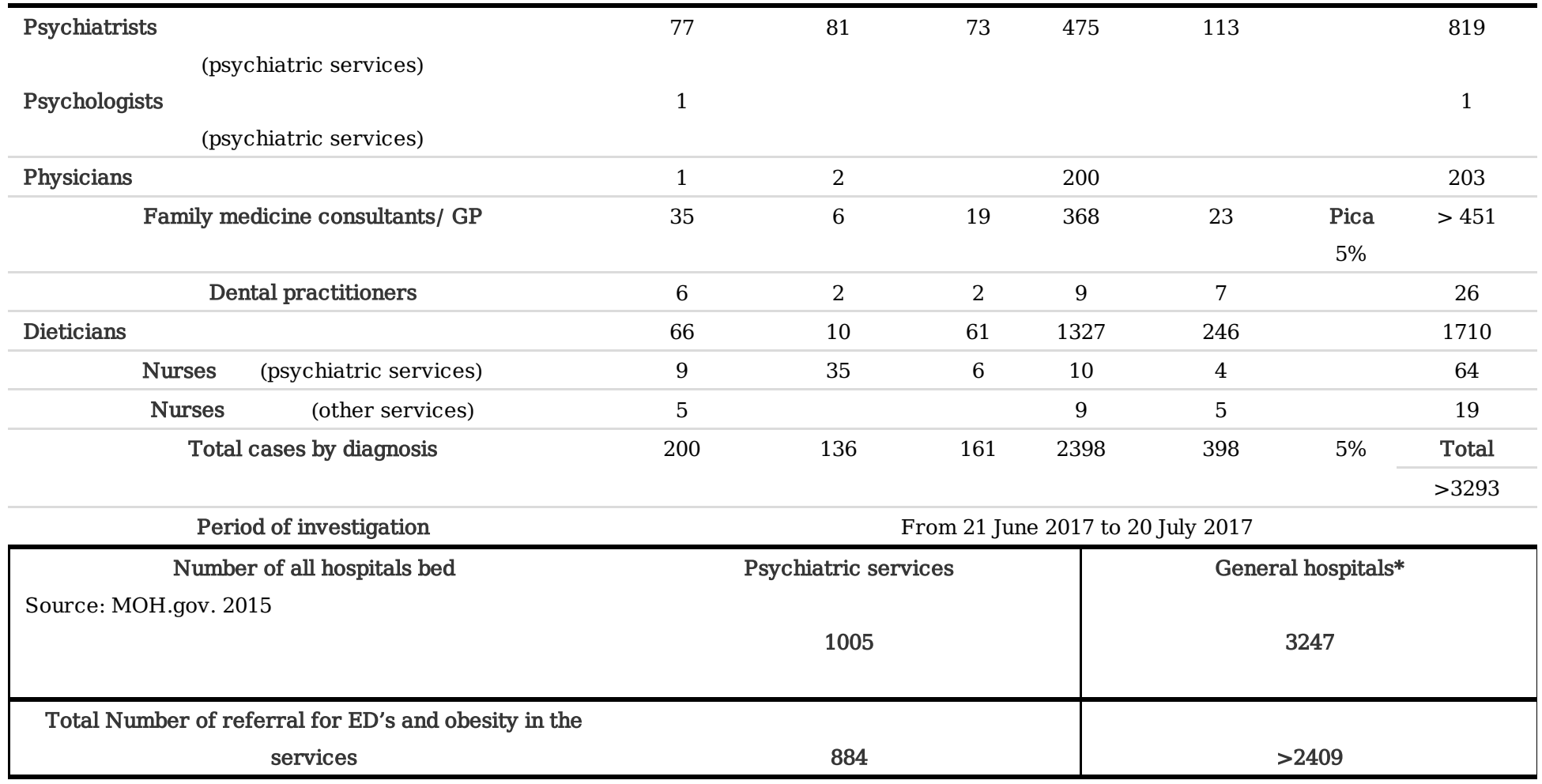

*General hospitals encompass services other than psychiatric services; general hospitals, specialised hospitals, medical complex and primary care.

Table 3: Proportions of professionals reported available treatment for all disorders in the services in \%. 


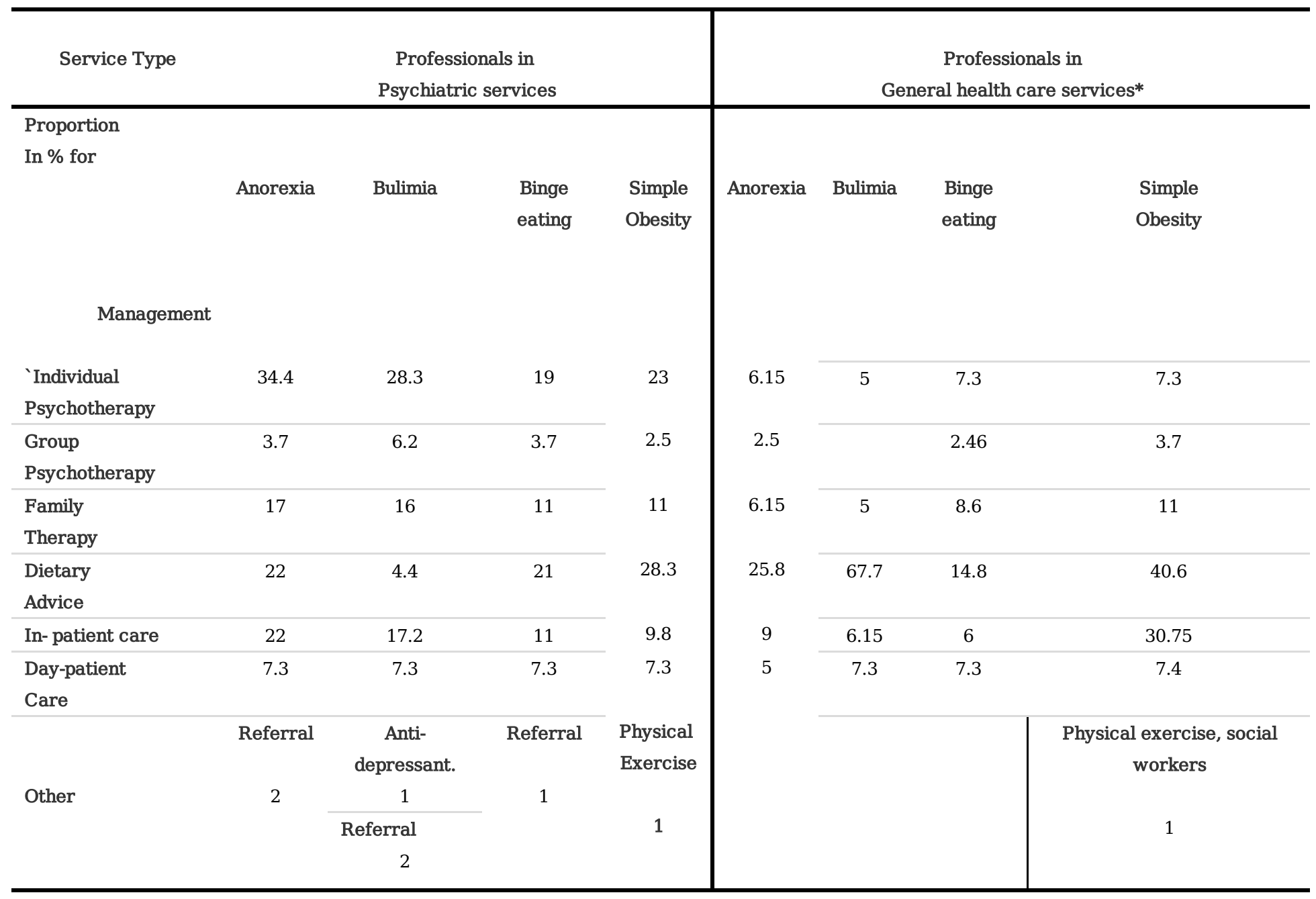

\begin{tabular}{|c|c|c|c|c|c|c|}
\hline $\begin{array}{l}\text { Care provision by } \\
\text { Eating disorders } \\
\text { specialists }\end{array}$ & $\begin{array}{c}\text { By } \\
\text { Eating disorders } \\
\text { specialists }\end{array}$ & $\begin{array}{l}\text { By generally } \\
\text { trained staff }\end{array}$ & $\begin{array}{c}\text { Not } \\
\text { answered }\end{array}$ & $\begin{array}{l}\text { Not specialised nor } \\
\text { generally trained }\end{array}$ & $\begin{array}{l}\text { Specialised ED's staff \& } \\
\text { generally trained staff }\end{array}$ & Total \\
\hline $\begin{array}{l}\text { Or by generally } \\
\text { trained staff }\end{array}$ & 5 & 89 & 28 & 7 & 1 & 123 \\
\hline
\end{tabular}

Table 4: The number of patients referred for bariatric surgery and BMI cut-offs.

\begin{tabular}{|c|c|c|}
\hline \multirow{3}{*}{ Obesity cases referred for bariatric surgery } & No. of cases & BMI cut offs \\
\hline & Median & Median \\
\hline & 4 & $>40$ \\
\hline
\end{tabular}

\section{Figures}




\section{Figure 1: The availability of ED specialists}

staff in psychiatric services

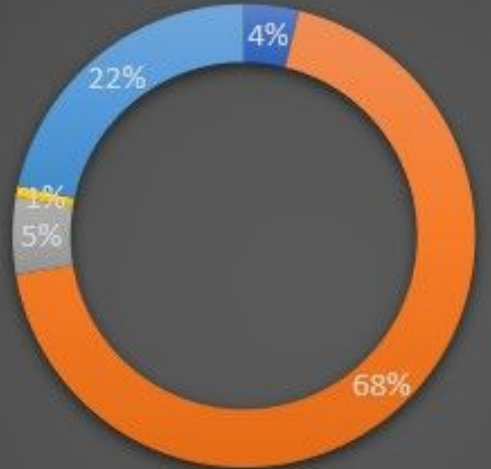

Eating disorders specialists

By generally trained staff

\section{Figure 1}

The availability of EDs specialists staff in psychiatric services. 